\title{
A WEAKER CONDITION FOR NORMALITY
}

\section{by IAN DOUST}

(Received 9 November, 1992)

1. Introduction. One of the most important results of operator theory is the spectral theorem for normal operators. This states that a normal operator (that is, a Hilbert space operator $T$ such that $T^{*} T=T T^{*}$ ), can be represented as an integral with respect to a countably additive spectral measure,

$$
T=\int_{\sigma(T)} \lambda d E(\lambda)
$$

Here $E$ is a measure that associates an orthogonal projection with each Borel subset of $\mathbb{C}$. The countable additivity of this measure means that if $x \in \mathscr{H}$ can be written as a sum of eigenvectors $x=\sum_{j=1}^{\infty} x_{j}$, then this sum must converge unconditionally.

It is of interest therefore to know when an operator is normal. One well-known characterization of normality is that $T$ have an isometric functional calculus for the continuous functions on the spectrum of $T, \sigma(T)$. That is there exists a $C^{*}$-algebra homomorphism $\psi: C(\sigma(T)) \rightarrow B(\mathscr{H})$ such that $\psi$ maps the polynomial $p(\lambda, \bar{\lambda})$ to $p\left(T, T^{*}\right)$. The details of this may be found in [5]. The aim of this paper is to show that the algebra $C(\sigma(T))$ may be replaced by a smaller algebra consisting of the absolutely continuous functions on some rectangle containing the spectrum of $T$.

This result is a special case of a condition which ensures that an operator on a reflexive $L^{p}$ space is scalar-type spectral. A scalar-type spectral operator on a Banach space is one which possesses the same type of spectral representation as that which the spectral theorem gives us for normal operators.

The corresponding result, showing that a Hilbert space operator which possesses a contractive functional calculus for the absolutely continuous operators on some compact interval of the real line is self-adjoint, was first proved by Fong and Lam in [6]. The extension of this to reflexive $L^{p}$ spaces appears in [2], where it is also shown that the existence of such a functional calculus does not imply that an operator on $L^{1}$ or $L^{\infty}$ is spectral.

2. Definitions. Throughout $\mathscr{H}$ will denote a separable complex Hilbert space, and $X$ will denote a complex Banach space. The set of bounded linear operators on $X$ is denoted by $B(X)$, and the set of all projections (that is, idempotent operators) on $X$ by $\operatorname{Proj}(X)$. Suppose that $\mathscr{F}$ is a Banach algebra of complex functions on some subset $S \subset \mathbb{C}$, and that the functions $e_{n}(z)=z^{n}$ lie in $\mathscr{F}$. Then by an $\mathscr{F}$ functional calculus we shall mean a Banach algebra homomorphism $\psi: \mathscr{F} \rightarrow B(X)$ such that $\psi\left(e_{n}\right)=e_{n}(T)=T^{n}, n=$ $0,1, \ldots$ A contractive functional calculus is one for which $\|\psi\|=1$.

An operator $T \in B(X)$ is said to be scalar-type spectral if there is a spectral measure $E$ defined on $\mathscr{B}$, the Borel subsets of $\mathbb{C}$, with values in $\operatorname{Proj}(X)$ such that

(i) $E$ is countably additive on $\mathscr{B}$ in the strong operator topology;

(ii) $T E(\Delta)=E(\Delta) T$, for all $\Delta \in \mathscr{B}$;

This work was supported by a grant from the Australian Research Council.

Glasgow Math. J. 36 (1994) 249-253. 
(iii) $\sigma(T \mid E(\Delta) X) \subset \bar{\Delta}$, for all $\Delta \in \mathscr{B}$;

(iv) $T=\int_{\sigma(T)} \lambda d E(\lambda)$.

On a Banach space which does not contain a copy of $c_{0}$, an operator $T$ is scalar-type spectral if and only if $T$ has a bounded $C(\sigma(T))$ functional calculus (see [5, Theorem 6.24] or [3, Proof of Theorem 2]). Thus scalar-type spectral operators may be regarded as a Banach space analogue of normal operators on a Hilbert space.

To develop a theory that includes operators whose spectral expansions may only converge conditionally, Smart [11] and Ringrose [10] introduced the notion of a well-bounded operator. This is an operator $T \in B(X)$ which possesses a functional calculus for the absolutely continuous functions on some compact interval of the real line. In other words, there exist $a, b, C \in \mathbb{R}$ such that

$$
\|g(T)\| \leq C\left\{|g(a)|+\int_{a}^{b}\left|g^{\prime}(t)\right| d t\right\}
$$

for all polynomials $g$. The spectral theorem for well-bounded operators on reflexive Banach spaces says that this is equivalent to $T$ having a spectral representation as an integral with respect to a 'spectral family' concentrated on the compact interval $[a, b] \subset \mathbb{R}$. Just as the spectral representation of a normal or a scalar-type spectral operator allows one to extend the functional calculus to include all bounded Borel measurable functions, the spectral representation for a well-bounded operator (on a reflexive space) allows one to extend its functional calculus to include all functions of bounded variation on $[a, b]$.

On a Hilbert space it is known that if the constant $K$ bounding the AC-functional calculus for $T$ can be chosen to be 1 , then $T$ must in fact be self-adjoint. The greatest difficulty in extending this result to cover normal operators, is the problem of deciding how to define functions of bounded variation in two variables. The correct definition in this context is the one due to Hardy [7] and Krause [8, p. 345]. Using this definition, Berkson and Gillespie generalized the concept of well-bounded operator to '.'clude operators whose spectrum may not be a subset of $\mathbb{R}$.

In the discussion that follows we shall identify subsets of $\mathbb{R}^{2}$ with subsets of $\mathbb{C}$ in the usual way. Let $J=[a, b]$ and $K=[c, d]$ be two compact intervals in $\mathbb{R}$. Let $\Lambda$ be a rectangular partition of $J \times K$ :

$$
a=s_{0}<s_{1}<\ldots<s_{n}=b, \quad c=t_{0}<t_{1}<\ldots<t_{m}=d .
$$

For a function $f: J \times K \rightarrow \mathbb{C}$, define

$$
V_{\Lambda}=\sum_{i=1}^{n} \sum_{j=1}^{m}\left|f\left(s_{i}, t_{j}\right)-f\left(s_{i}, t_{j-1}\right)-f\left(s_{i-1}, t_{j}\right)+f\left(s_{i-1}, t_{j-1}\right)\right|
$$

The variation of $f$ is defined to be

$$
\underset{J \times K}{\operatorname{var} f}=\sup \left\{V_{\Lambda}: \Lambda \text { is a rectangular partition } J \times K\right\} .
$$

We shall say that the function $f$ is of bounded variation if $\underset{J \times K}{\operatorname{var} f,} \underset{J}{\operatorname{var}} f(\cdot, d)$, and 
$\underset{K}{\operatorname{var}} f(b,$.$) are all finite. The set \mathrm{BV}(J \times K)$ of all functions $f: J \times K \rightarrow \mathbb{C}$ of bounded variation is a Banach algebra under the norm

$$
\|f\||=| f(b, d) \mid+\underset{J}{\operatorname{var}} f(\cdot, d)+\underset{K}{\operatorname{var}} f(b, \cdot)+\underset{J \times K}{\operatorname{var}} f .
$$

As with functions of one variable, there is the concept of an absolutely continuous function. Let $m$ denote Lebesgue measure on $\mathbb{R}^{2}$. A function $f: J \times K \rightarrow \mathbb{C}$ is said to be absolutely continuous if

(i) for all $\varepsilon>0$, there exists $\delta>0$ such that

$$
\sum_{R \in \mathscr{R}} \operatorname{var}_{R} f<\varepsilon
$$

whenever $\mathscr{R}$ is a finite collection of non-overlapping subrectangles of $J \times K$ with

$$
\sum_{R \in \mathscr{R}} m(R)<\delta
$$

(ii) the marginal functions $f(\cdot, d)$ and $f(b, \cdot)$ are absolutely continuous functions on $J$ and $K$ respectively.

The set $\mathrm{AC}(J \times K)$ of all absolutely continuous functions $f: J \times K \rightarrow \mathbb{C}$ is a Banach subalgebra of $\mathrm{BV}(J \times K)$, and is the closure in $\mathrm{BV}(J \times K)$ of the polynomials in two real variables on $J \times K$. Equivalently, one can consider $\mathrm{AC}(J \times K)$ to be the closure of the polynomial functions $p(z, \tilde{z})$ on $J \times K \subset \mathbb{C}$. It is clear that $\mathrm{AC}(J \times K) \subset C(J \times K)$ and that $\|f\|_{\infty} \leq\|f\|$ for all $f \in \mathrm{AC}(J \times K)$. The full details of these spaces can be found in [1].

An operator $T \in B(X)$ is said to be an $A C$-operator if there exists a Banach algebra homomorphism $\psi: \mathrm{AC}(J \times K) \rightarrow B(X)$ for which $\psi(z \mapsto z)=T$. Berkson and Gillespie proved that this is equivalent to the condition that $T$ can be written as $T=U+i V$, where $U$ and $V$ are commuting well-bounded operators on $X$.

By $L^{p}$ we shall mean the usual Lebesgue space of complex valued, $p$-integrable functions (or essentially bounded functions in the case that $p=\infty$ ), on some positive measure space.

3. The results. In this section we shall show that on the reflexive $L^{\prime \prime}$ spaces, the apparently weaker condition of having a contractive $\mathrm{AC}(J \times K)$ functional calculus, actually implies that $T$ has a bounded $C(J \times K)$ functional calculus.

THEOREM 3.1. Suppose that $1<p<\infty$ and that $T$ is a bounded operator on $L^{p}$. Then, if there exist compact intervals $J, K \subset \mathbb{R}$ such that $T$ has a contractive $\mathrm{AC}(J \times K)$ functional calculus, then $T$ is a scalar-type spectral operator.

Proof. Suppose that $T$ has a contractive $\mathrm{AC}(J \times K)$ functional calculus. Then $T$ can be written as $T=U+i V$ for a unique pair of well-bounded operators. Suppose that $h \in \mathrm{AC}(J)$. Then the function defined by $g(s+i t)=h(s)$ lies in $\mathrm{AC}(J \times K)$ and $g(T)=$ $h(U)$. Note that $\|h\|_{\mathrm{AC}(J)}=\|\| g \|_{\mathrm{AC}(J \times K)}$, so that

$$
\|h(U)\|=\|g(T)\| \leq\|g\|_{\mathrm{AC}(J \times K)}=\|h\|_{\mathrm{AC}(J)} .
$$

By [2]; this implies that $U$ is a scalar-type spectral operator on $L^{p}$. Similar reasoning implies that $V$ (and hence $i V$ ) is also a scalar-type spectral operator. It follows by a result 
of McCarthy [9] that the sum of two commuting scalar-type spectral operators on a reflexive $L^{p}$ space is also a scalar-type spectral operator. Hence $T$ is scalar-type spectral.

Given an AC-operator $T=U+i V$ acting on a reflexive $L^{p}$ space, we can define its conjugate operator to be $\bar{T}=U-i V$. Note that on a reflexive Banach space $U$ and $V$ are uniquely determined, so $\bar{T}$ is well-defined. Whether this splitting into the 'real and imaginary parts' of a general AC-operator is unique appears to be an open question.

Corollary 3.2. Suppose that $1<p<\infty$ and that $T$ is an AC-operator on $L^{p}$. Suppose also that for all polynomials in $z$ and $\bar{z}$ on $J \times K \subset \mathbb{C},\|g(T, \bar{T})\| \leq\|g\|_{\mathrm{AC}(J \times K)}$. Then there exists $C \in \mathbb{R}$ such that $\|g(T, \bar{T})\| \leq C\|g\|_{C(\sigma(T))}$ for all such $g$.

In Hilbert spaces we can show that these functional calculus conditions are equivalent.

THEOREM 3.3. Suppose that $T$ is a bounded operator on a Hilbert space $\mathscr{H}$. Then the following conditions are equivalent:

(i) $T$ is normal;

(ii) $T$ possesses an isometric $C(\sigma(T))$ functional calculus;

(iii) $T$ possesses a contractive functional calculus for the bounded Borel measurable functions on $\sigma(T)$;

(iv) there exist compact intervals $J, K \subset \mathbb{R}$ such that $T$ has a contractive $C(J \times K)$ functional calculus;

(v) there exist compact intervals $J, K \subset \mathbb{R}$ such that $T$ has a contractive $\mathrm{BV}(J \times K)$ functional calculus;

(vi) there exist compact intervals $J, K \subset \mathbb{R}$ such that $T$ has a contractive $\mathrm{AC}(J \times K)$ functional calculus.

Proof. The equivalence of the first four conditions is standard. To show that (iii) $\Rightarrow(\mathrm{v})$, it suffices to note that for $f \in \mathrm{BV}(J \times K)$,

$$
\|f(T)\| \leq\|f\|_{\mathscr{B}(\sigma(T))} \leq\|f\|_{\mathscr{B}(J \times K)} \leq^{-}\|f\|_{\mathrm{BV}(J \times K)} .
$$

That $(v) \Rightarrow(v i)$ is trivial. To show that (iv) $\Rightarrow$ (i), one can proceed as in Theorem 3.1. In this case the existence of a contractive $A C(J)$ functional calculus for $U$ implies that $U$ is self-adjoint (see [5], [4]). It is easy to check that if $U$ and $V$ are commuting self-adjoint operators, then $T=U+i V$ is normal.

It is interesting to note that in general, it is not known whether an AC-operator on a reflexive Banach space (or more generally, an operator on any Banach space which can be written as $T=U+i V$ with $U$ and $V$ commuting well-bounded operators of type (B)), must have a $\operatorname{BV}(J \times K)$ functional calculus.

\section{REFERENCES}

1. E. Berkson and T. A. Gillespie, Absolutely continuous functions of two variables and well-bounded operators, J. London Math. Soc. (2) 30 (1984), 305-321.

2. I. Doust, Well-bounded and scalar-type spectral operators on $L^{p}$ spaces, J. London Math. Soc. (2) 39 (1989), 525-534.

3. I. Doust, Well-bounded and scalar-type spectral operators on spaces not containing $c_{0}$, Proc. Aimer. Math. Soc. 105 (1989), 367-370. 
4. 1. Doust, An example in the theory of spectral and well-bounded operators, Miniconference on Operators in Analysis (Sydney, 1989), Proc. Centre Math. Anal. Austral. Nat. Univ. 24 (1990), 83-90.

5. H. R. Dowson, Spectral theory of linear operators, London Mathematical Society Monographs 12 (Academic Press, 1978).

6. C. K. Fong and L. Lam. On spectral theory and convexity, Trans. Amer. Math. Soc. 264 (1981), 59-75.

7. G. H. Hardy, On double Fourier series, Quart. J. Math. 37 (1906), 53-79.

8. E. W. Hobson, The theory of functions of a real variable and the theory of Fourier's series, Vol. 1, third edition (Dover, 1957).

9. C. A. McCarthy, Commuting Boolean algebras of projections II, Proc. Amer. Math. Soc. 15 (1964), 781-787.

10. J. R. Ringrose, On well-bounded operators, J. Austral. Math. Soc. 1 (1959/60), 334-343.

11. D. R. Smart, Conditionally convergent spectral expansions, J. Austral. Math. Soc. 1 $(1959 / 60), 319-333$.

School of Mathematics

UNIVERSiTY OF NeW SOUTH Wales

KENSINGTON

NSW 2033

AUSTRALIA 